\author{
CANADIAN $\neq$ THORACIC SOCIETY \\ SOCIÉTÉ T. CANADIENNE DE THORACOLOGIE
}

\section{A new chapter}

S ince the last issue of the Journal, the Canadian Thoracic Society (CTS) Board and Executive have been working collaboratively with the leadership of the Canadian Lung Association (CLA) to establish the CTS as a distinct legal entity. The move to becoming an independent organization is a logical evolution given how the Society has matured, and how its role and mandate has expanded over time.

A new milestone for the CTS was achieved on Wednesday August 5, 2015, when we received our approved articles of incorporation from Industry Canada!

In the lead-up to this historical moment, we have received legal counsel on all of the required steps to develop the appropriate structure, bylaws and operating policies. I am pleased to report that all of the current members of the CTS Board have confirmed their commitment to serve as Directors of the new corporation. In alignment with our partnership with CLA and historical practice, we have also made provisions to appoint a representative from the Lung Association to the CTS Board of Directors.

Our goal in this evolutionary process is for both CLA and CTS to be stronger, and for our impact on lung health to grow significantly. We are committed to working together to ensure that happens. For example, the CLA and CTS will continue to work together on key initiatives such as the National Respiratory Research Strategy and the Breathing as One national fundraising campaign for research (www. BreathingasOne.ca). The Canadian Respiratory Conference will also continue to be a partnership initiative of the CTS, CRHP and the CLA (https://crc.lung.ca).

Being incorporated allows the CTS to manage its financial and administrative affairs independently of the CLA, and to focus specifically on our mandate as a professional society. We will be operating in a similar fashion to the Canadian Cardiovascular Society and the Heart and Stroke Foundation, who share office space and collaborate on common goals, but deliver on very distinct mandates to the health care community and the Canadian public, respectively.

While we have been immersed in the legal and administrative requirements of incorporation, we are working diligently to maintain the momentum of our strategic agenda by:

- Fostering innovation to promote evidence-based clinical practice;

- Providing state-of-the art professional education to transform lung health care;

- Fostering cutting edge lung health research; and

- Developing the next generation of respiratory health professionals and researchers.

We are striving to facilitate a smooth transition over the coming months. While all programs and deliverables are moving forward as planned, there have been a few understandable delays in signing corporate sponsorship contracts as part of due process. I would like to acknowledge, with deep appreciation, the patience of our industry partners as we have made the transition in financial management. We are looking forward to the first meeting of the CTS Industry Advisory Council in September, where we will be able to update corporate sponsors on progress to date and to identify areas for further collaboration.

CTS members - stay tuned for further news and developments! New opportunities to enhance member benefits and increase collaboration are emerging in discussions with our international partners such as the American Thoracic Society, CHEST and the European Respiratory Society. In addition to updates provided through our

\section{Un nouveau chapitre}

$\mathrm{D}_{\mathrm{s}}$ epuis la parution du dernier numéro de la Revue canadienne de pneumologie, le conseil d'administration et le comité exécutif de la Société canadienne de thoracologie (SCT) collaborent avec la direction de l'Association pulmonaire du Canada (APC) à établir la SCT en tant que personne morale distincte. Cette démarche visant à devenir un organisme indépendant est une mesure logique considérant l'évolution de la SCT et l'élargissement de son rôle et de son mandat au fil du temps.

Le mercredi 5 août 2015 a marqué un nouveau jalon pour la SCT : la réception de ses statuts constitutifs approuvés par Industrie Canada!

Avant ce moment historique, nous avions reçu des conseils juridiques sur les mesures à prendre pour développer la structure, les règlements et les politiques opérationnelles appropriés. Je suis heureuse d'annoncer que tous les membres actuels du conseil d'administration de la SCT ont confirmé leur engagement à poursuivre leur mandat au sein de la nouvelle corporation. Conformément à notre partenariat avec l'APC et à nos pratiques antérieures, nous avons également inclus une disposition visant à nommer un représentant de l'Association pulmonaire au conseil d'administration de la SCT.

Dans le cadre de ce processus évolutif, nos objectifs sont de renforcer l'APC et la SCT et d'accroître considérablement notre impact sur la santé pulmonaire. Nous sommes engagés à travailler ensemble à l'atteinte de ces objectifs. Par exemple, l'APC et la SCT continueront de collaborer à des initiatives clés comme la Stratégie nationale de recherche en santé respiratoire et la campagne nationale de collecte de fonds pour la recherche Un monde, un souffle (www. BreathingasOne.ca). Le Congrès canadien sur la santé respiratoire demeurera également une initiative de partenariat entre la SCT, les PCSR et l'APC (https://crc.lung.ca).

Le fait d'être constituée en personne morale permet à la SCT de gérer ses affaires financières et administratives indépendamment de l'APC et de se concentrer plus précisément sur son mandat de société professionnelle. Nous fonctionnerons de manière similaire à la Société canadienne de cardiologie et à la Fondation des maladies du cœur et de l'AVC, qui partagent des locaux et collaborent à des buts communs, mais remplissent des mandats très distincts auprès de la communauté des soins de la santé et du public canadien, respectivement.

Alors que nous sommes plongés dans les exigences juridiques et administratives de la constitution en personne morale, nous travaillons fort à maintenir l'élan de notre ordre du jour stratégique par les actions suivantes :

- appuyer l'innovation pour promouvoir des pratiques cliniques fondées sur des données probantes;

- offrir une formation professionnelle de pointe pour transformer les soins de santé pulmonaire;

- favoriser des recherches d'avant-garde en santé pulmonaire; et

- former la prochaine génération de professionnels et de chercheurs en santé respiratoire.

Nous nous efforcerons de favoriser une transition en douceur, au cours des prochains mois. Même si tous les programmes et livrables progressent comme prévu, nous avons rencontré quelques retards compréhensibles dans la signature des contrats de commandites d'entreprises, vu les procédures établies. Je remercie sincèrement nos partenaires de l'industrie pour leur patience dans la phase de transition de notre gestion financière. Nous attendons avec impatience la première réunion du Comité consultatif de la SCT sur l'industrie, en septembre, 
member e-bulletin and website, we hope to have an opportunity at the upcoming CHEST conference in Montreal (Quebec) to provide attending members with more detailed information. Be sure your membership is current so that you receive an invitation to the annual CTS reception at CHEST!

Ensuring the viability and vitality of our growing society will continue to be a significant focus for the CTS Executive and Board of Directors in the months ahead. We greatly appreciate your ongoing commitment to the CTS and your participation in the work of the Society. I invite you to renew your membership (and encourage your colleagues to do the same), attend the Canadian Respiratory Conference, and contribute to our endeavours to improve lung health for all Canadians!

Respectfully submitted, Diane Lougheed MD MSc FRCP President, Canadian Thoracic Society lors de laquelle nous informerons nos sociétés commanditaires des progrès à ce jour et identifierons des domaines de collaboration ultérieure.

Chers membres de la SCT, surveillez les annonces et nouveautés à venir! Des occasions de bonifier les avantages des membres et de rehausser la collaboration émergent de nos discussions avec des partenaires internationaux comme l'ATS, CHEST et l'ERS. En plus des mises à jour que nous publions dans notre bulletin électronique et sur notre site Internet, nous espérons fournir des informations encore plus détaillées à nos membres lors du Congrès CHEST à Montréal. Assurez-vous que votre adhésion à la SCT est en bonne et due forme afin de recevoir une invitation à la réception annuelle de la SCT au Congrès CHEST!

La viabilité et la vitalité de notre société grandissante demeureront d'importants points de mire du comité exécutif et du conseil d'administration de la SCT au cours des prochains mois. Nous apprécions grandement votre appui continu à la SCT et votre participation à son travail. Je vous invite à renouveler votre adhésion (et j'encourage vos collègues à en faire autant), à participer au Congrès canadien sur la santé respiratoire et à contribuer à nos initiatives pour améliorer la santé pulmonaire de tous les Canadien-nes!

Le tout respectueusement soumis, Diane Lougheed MD, M. Sc., FRCP Présidente, Société canadienne de thoracologie 


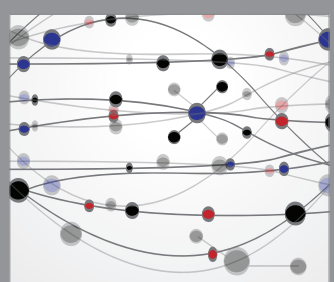

The Scientific World Journal


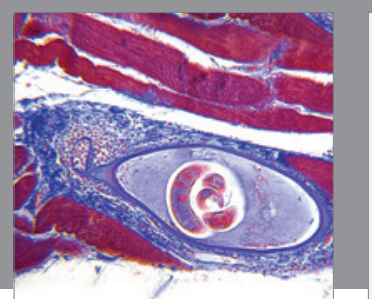

Gastroenterology Research and Practice

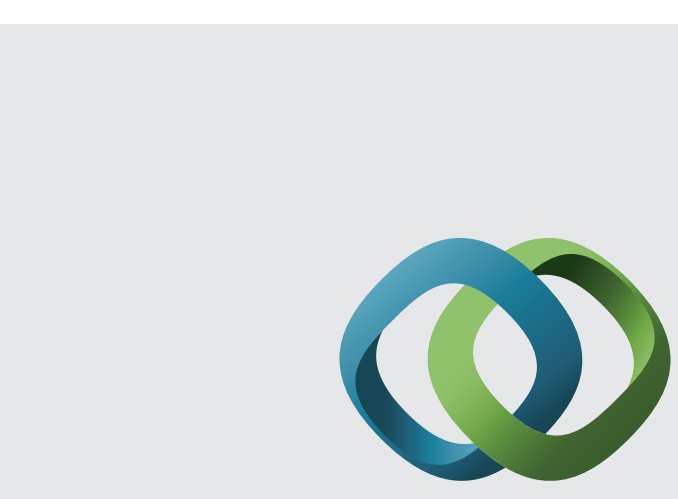

\section{Hindawi}

Submit your manuscripts at

http://www.hindawi.com
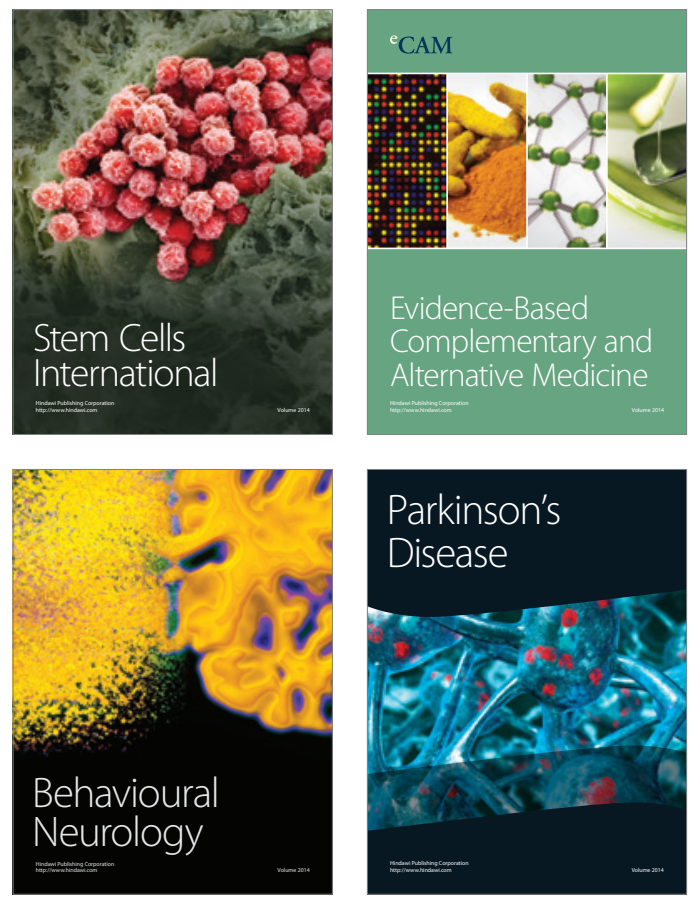
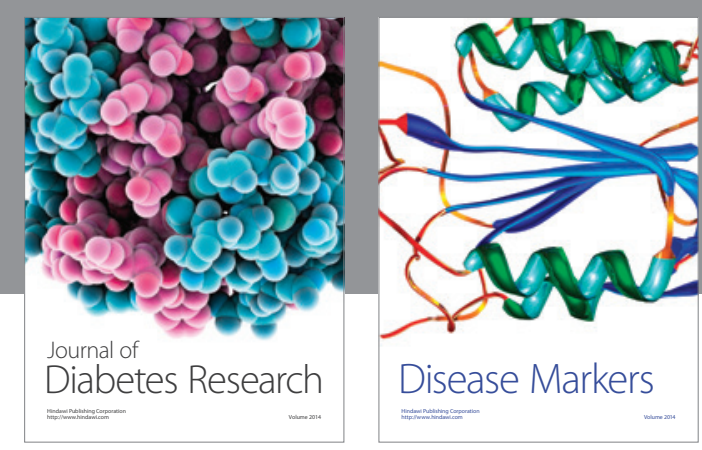

Disease Markers
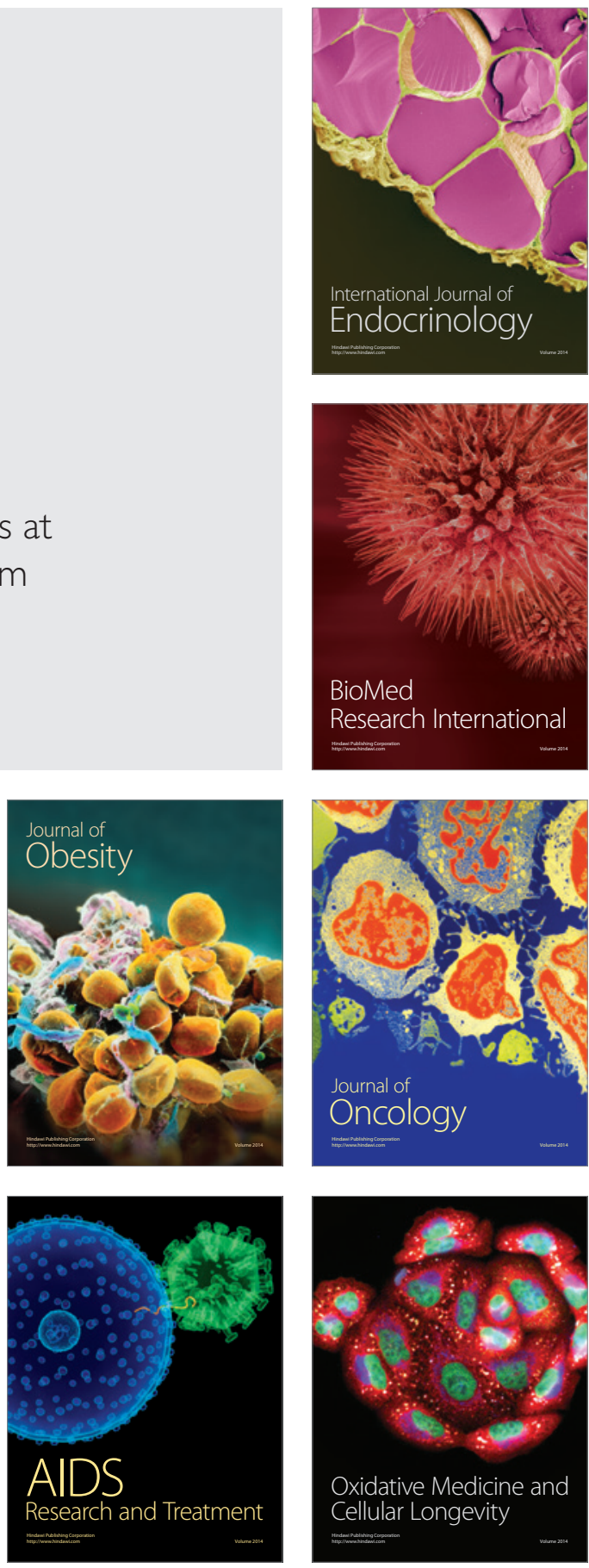